Original

\title{
A Coronary Primary Intervention Study of Japanese Men : Study Design, Implementation and Baseline Data
}

\author{
The Kyushu Lipid Intervention Study Group
}

Members of the Study Group are listed in Appendix 1

\begin{abstract}
This report describes the design and baseline results of the Kyushu Lipid Intervention Study (KLIS). The study aims to test the hypothesis that the long-term reduction of serum total cholesterol by pravastatin will lead to a decrease in coronary heart disease (CHD) events. The trial was designed to include a random 6,000 male patients aged 45-74 years with serum total cholesterol of $220 \mathrm{mg} / \mathrm{dl}(5.69 \mathrm{mmol} / \mathrm{l})$ or greater and without a history of myocardial infarction, coronary surgery or angioplasty, to undertake either pravastatin or conventional treatment (including hypolipidemic drugs other than HMG-CoA reductase inhibitors, probucol and bezafibrate), and to follow up each patient for 5 years. Primary endpoints are fatal and nonfatal myocardial infarction, coronary bypass surgery and angioplasty, cardiac death, and sudden and unexpected death. During the period from May 1990 to September 1993, a total of 5,640 male patients aged 45-74 were recruited by 902 participating physicians throughout Kyushu. Randomization was, however, neglected by study physicians; the numbers of patients enrolled were 3,061 in the pravastatin group and 2,579 in the conventional treatment group. Patients allocated to the pravastatin treatment were generally unfavorable regarding coronary risk factors. Baseline mean levels of serum total cholesterol were $259 \mathrm{mg} / \mathrm{dl}(6.70 \mathrm{mmol} / \mathrm{l})$ in the pravastatin group and $246 \mathrm{mg} / \mathrm{dl}$ $(6.36 \mathrm{mmol} / \mathrm{l})$ in the conventional treatment group $(p<0.001)$. Although the trial was regarded as a prospective observational study, the KLIS provides valuable quantitative data regarding cholesterol lowering and reduction in CHD events as well as safety data of the long-term use of a statin in Japanese men with hypercholesterolemia. $J$ Atheroscler Thromb, 1996 ; 3 : 95-104.
\end{abstract}

Key words : Primary prevention trial, Design, Hypercholesterolemia, Coronary heart disease, Pravastain, Japanese men

Elevated blood cholesterol levels have consistently been associated with an increased risk of coronary heart disease (CHD) in many prospective studies, but almost exclusively in Western countries (1-4). Intervention trials also have reported that the use of cholesterol-lowering drugs is beneficial in preventing the occurrence of CHD among hypercholesterolemic subjects. In the WHO clofibrate trial, a $9 \%$ reduction in plasma total cholesterol was associated with a $20 \%$ decrease in cardiac endpoints

Address for correspondence: Professor Kikuo Arakawa, MD, The Second Department of Internal Medicine, Fukuoka University School of Medicine, Jonan-ku, Fukuoka 814-80, Japan.

Received March 15, 1996.

Accepted for publication October 15, 1996. during a 5-year follow-up period $(5,6)$. The Lipid Research Clinic (LRC) trial observed a $19 \%$ decrease in cardiac endpoints over a 7 -year period associated with a $8 \%$ fall in plasma total cholesterol among men receiving cholestyramine $(7,8)$. In addition, the Helsinki Heart Study observed an $11 \%$ fall in plasma total cholesterol and a $34 \%$ reduction in cardiac deaths, and fatal and nonfatal myocardial infarction combined over a 5-year period in the gemfibrozil-treated group as compared with the placebo group (9). These intervention trials, however, have failed to show a reduction in total mortality in subjects receiving lipid-lowering drugs; and even an excess mortality was observed in the drug-treated group in the WHO trial $(5,6)$.

Clinical practice for hypercholesterolemia in Japan is 
basically guided by data of Western populations with much higher risks of CHD. Although a prospective study of atomic bomb survivors in Japan has reported a significant, positive association between plasma total cholesterol and CHD incidence (10), it remains uncertain whether the use of lipid-lowering drugs is beneficial for hypercholesterolemic patients in Japan. It has been difficult to conduct a coronary prevention trial of lowering of cholesterol in Japan, where the mortality rate of CHD is the lowest among industrialized countries. The development of 3-hydroxy-3-methylglutaryl coenzyme A (HMG$\mathrm{CoA})$ reductase inhibitors, powerful cholesterol-lowering drugs $(11,12)$, resulted in the opportunity to perform such a trial even in Japan. The Kyushu Lipid Intervention Study (KLIS), using pravastatin or the prototype of HMG-CoA reductase inhibitors, was designed to obtain quantitative information on the reduction in serum total cholesterol and $\mathrm{CHD}$ risk as well as data on the safety of the long-term use of this newly introduced drug in Japanese men. Recently, a randomized trial using pravastatin in the West of Scotland reported not only an evident decrease in CHD events but also a nearly significant reduction in all-cause mortality among treated patients (13). The present paper describes methodological aspects of the KLIS and discusses the rationale for continuing the study.

\section{Study Design}

\section{General design}

The KLIS is a primary prevention trial designed to test a hypothesis that pravastatin, compared with the conventional regimen (see below), will be more effective in reducing coronary mortality and morbidity in Japanese male patients aged 45-74 years with primary hypercholesterolemia defined as $220 \mathrm{mg} / \mathrm{dl}(5.69 \mathrm{mmol} / \mathrm{l})$ or greater. The study is a randomized controlled trial, in which each patient is treated and followed up for 5 years within the framework of the routine medical care system. Treatment is not blind either to patients or to study physicians. This policy is taken so as to attenuate the pressure on the side of participating physicians, and for them to detect promptly any possible adverse effects of the drugs. Since physicians generally have a strong idea that they should provide a certain type of treatment, preferably pharmacological therapy, to their patients, it is not feasible to use a placebo group. The study was designed in accordance with the Helsinki Declaration on medical research, and was approved by the Ethical Committee of Fukuoka University School of Medicine.

\section{Participants}

Study subjects are recruited among patients visiting physicians participating in the study. Eligible patients are men aged $45-74$ years with serum total cholesterol of $220 \mathrm{mg} / \mathrm{dl}(5.69 \mathrm{mmol} / \mathrm{l})$ or greater at two occasions in the prerandomization phase. The definition of hypercholes- terolemia is in accordance with the guideline of the Japan Atherosclerosis Association (14).

Men with serum high-density lipoprotein (HDL) cholesterol of $80 \mathrm{mg} / \mathrm{dl}(2.07 \mathrm{mmol} / \mathrm{l})$ or greater are not eligible. Also excluded are men with a history of myocardial infarction, coronary bypass surgery, coronary angioplasty, cerebral hemorrhage or cerebral infarction; those with congenital or rheumatic heart disease; those with significantly life-limiting conditions such as renal failure and liver disease ; those with drug sensitivity ; and those with conditions associated with secondary hyperlipidemia such as nephrotic syndrome, hypothyroidism and steroid use. Angina pectoris and diabetes mellitus are not exclusion criteria; the presence of angina pectoris is based on a physician's reported diagnosis, while diabetes mellitus is determined either when hemoglobin A1c is $6.5 \%$ or greater or when fasting blood sugar is $140 \mathrm{mg} / \mathrm{dl}$ or higher.

Each eligible patient is informed of the study purpose, medication methods, possible beneficial and adverse effects, and rights of voluntary withdrawal from the study. Potential participants not under medication for hyperlipidemia are observed for at least 2 weeks ; patients on hypolipidemic drugs are observed for at least 4 weeks after discontinuation of the lipid-lowering drugs.

\section{Endpoints}

The major endpoints of the study are fatal and nonfatal myocardial infarction, coronary artery surgery and angioplasty, cardiac death, and sudden and unexpected death. Detailed definitions of these events are described in Appendix 2. The combined incidence of these coronary events is a primary outcome of the study. Outcomes of secondary interest'are cerebral infarction and total mortality. Transient cerebral ischemia is not considered as an outcome variable. Diagnostic criteria for cerebral infarction are described in Appendix 3.

\section{Sample size}

The study population is 6,000 men randomized equally to either the pravastatin or conventional treatment. The required sample size was calculated to be 2,866 in each group using a normal approximation to the binominal distribution (15). Because of crude estimations of various parameters, a slightly larger sample size was set as a target of patient recruitment. The $5 \%$ significance level (one-sided) and the $20 \%$ beta level (power of the study= $80 \%$ ) were chosen. A $20 \%$ drop-out rate in five years was assumed in both treatment groups, but the length of time required to achieve maximum benefit of treatment was not taken into account (15). The combined incidence rate of coronary events in the cohort was expected to be $3.5 \%$ for the 5 years without any treatment, based on several assumptions, as described in detail in Appendix 4 (16-21)

Reduction in serum total cholesterol was projected to 
be $5 \%$ in the conventional treatment group and $15 \%$ in the pravastatin group. With reference to data from the WHO and LRC trials (5-8), the reduction of the combined CHD endpoint was expected to be at most $10 \%$ in the conventional treatment group. Since pravastatin has been shown to have beneficial effects on HDL cholesterol and triglycerides $(11,12)$, a $45 \%$ reduction in $\mathrm{CHD}$ events was assumed in the pravastatin group, taking into account the results of the Helsinki Heart Study (9).

\section{Randomization}

Randomization is carried out by using a set of 4 sealed envelopes by which 4 patients are randomly and equally allocated either to pravastatin or to conventional treatment. A set or sets of sealed envelopes are distributed to each participating physician, and the envelopes are to be opened in numerical order after confirmation of eligibility of patients and their voluntary participation.

\section{Intervention}

Pravastatin is prescribed at a dosage of $10-20 \mathrm{mg}$ per day. This prescription is officially approved, and has shown to result in a $15-20 \%$ reduction in serum total cholesterol on average in hypercholesterolemic patients $(11,12)$. The conventional treatment includes medication with hypolipidemic drugs other than probucol, bezafibrate and simvastatin as well as dietary and/or exercise therapy. The dosages of hypolipidemic drugs are determined by each physician in accordance with prescriptions approved by the Ministry of Health and Welfare. Participating physicians are not given any specific instructions regarding nonpharmacological treatment to their patients.

\section{Follow-up}

Enrolled patients in both groups are medically cared for by the study physicians. Since the approved length of prescription for hyperlipidemia is maximally 4 weeks, patients generally visit their physicians every 4 weeks. Physicians are requested to report promptly the cardiovascular endpoints and all deaths to the Trial Office. Periodic follow-up forms are sent to each physician at 3 , 6 , and 12 months after the beginning of treatment, and annually thereafter for the purpose of obtaining clinical and laboratory data on adverse effects as well as completed reports of the endpoints. When an endpoint is reported, an additional survey form is sent to each physician to collect detailed relevant data. Deaths are to be monitored after the occurrence of endpoints and also after the 5-year of treatment or discontinuation of the treatment.

\section{Study Organization}

\section{Functional unit}

Trial Office: The Trial Office is based at the Second Department of Internal Medicine, Fukuoka University
School of Medicine. The Office is responsible for general study coordination, and plays a central function in the conducting of the trial. The Office recruits physicians participating in the study with help of local study organizers (key persons in areas or districts) throughtout Kyushu. Sealed envelopes for randomization, entry forms, baseline survey forms, and periodic follow-up forms are directly sent to each participating physician by the Trial Office. All data entry and validation of case report forms are initially carried out at the Office, and sent to the Data Management Section for computerization.

The Data Management Section is a subsidiary unit of the Trial Office, and is also housed at the Second Department of Internal Medicine, Fukuoka University School of Medicine. Data management is supported by computer technicians and statisticians from external institutions. This section is in charge of analyzing and storing all data, and prepares statistical reports of the trial results for the External Review Committee and appropriate data for the internal committees.

Management and committees (Appendix 1 for membership)

Executive Committee: The committee is the primary management group for the study. This group formulates policy and directs various activities in conducting the study. The decisions made by the Committee are, however, to be approved or amended at the annual meeting of the area physician organizers, who represent area-based groups of study physicians. Two subcommittees are placed under the Executive Committee in order to evaluate biannually or more frequently cardiovascular endpoints and adverse effects and to give appropriate instructions on data analysis.

Cardiovascular Endpoint and Adverse Effect Subcommittee: This working group includes two certified cardiologists and one certified neurologist. They are in charge of classifying cardiovascular endpoints. The subcommittee blindly validates reported cases of cardiovascular events by reviewing abstracts of medical records prepared by the study physicians, laboratory data and serial electrocardiograms (ECG). The underlying cause of death is also classified and coded in accordance with the 9th revision of the International Disease Classification (ICD), based on a reported abstract of the medical record. This subcomittee also blindly reviews and scrutinizes notified adverse effects based on clinical and laboratory data, and provides advice as to treatment and patient's withdrawal, to the study physicians when necessary. This group also monitors the occurrence of any severe diseases including cancer and cerebral hemorrhage based on disease conditions recorded on the periodic follow-up forms. Additional, detailed information on diseases are obtained from the study physicians when needed.

Data Analysis Subcomittee: This subcomittee consists 
of an expert statistician and an epidemiologist, and provides detailed instruction to the Data Management Section of the Trial Office regarding data analysis. Any statistical issues in the trial are settled by this subcommittee.

External Review Committee: This group is an advisory committee to the Executive Committee set up to ensure the safety of patients and the integrity of the trial. The committee reviews the study protocol, conduct of the trial, and reported data on adverse effects and cardiovascular endpoints arising from the interim and final analyses. Data on adverse effects (including mortality data) are subject to annual evaluation. An interim analysis on the primary endpoints is planned in the middle of the followup period, and the Committee makes a recommendation as to stoppage, continuation, or extension of the trial.

\section{Conduct of the Trial}

\section{Enrollment}

Each participating physician was responsible for enrollment of eligible patients, acquisition of informed consent, and random allocation using the sealed envelopes. During the period from May 1990 to September 1993, including a 10-month period of pilot study, a total of 902 physicians recruited 5,640 male patients fulfilling the eligibility criteria (Table 1 ).

\section{Baseline survey and laboratory measurements}

At the prerandomization phase, besides the measure-

Table 1. Number of study physicians according to number of patients recruited.

\begin{tabular}{cc}
$\begin{array}{c}\text { Number of recruited patients } \\
\text { per physician }\end{array}$ & Number of physicians \\
\hline 1 & 167 \\
2 & 153 \\
3 & 118 \\
4 & 109 \\
5 & 52 \\
6 & 43 \\
7 & 42 \\
8 & 39 \\
9 & 33 \\
10 & 19 \\
11 & 18 \\
12 & 13 \\
13 & 11 \\
14 & 10 \\
15 & 10 \\
16 & 8 \\
17 & 8 \\
18 & 5 \\
19 & 5 \\
$20+$ & 39
\end{tabular}

ments of serum total cholesterol, HDL cholesterol and triglycerides, other clinical and laboratory data were obtained (see Appendix 5). Also recorded at the baseline survey were prior use of hypolipidemic drugs, concurrent medication, and prevalent diseases, smoking habit and alcohol intake frequency.

Laboratory measurements including the determination of serum total cholesterol were carried out at different laboratories, but each physician was asked to use the same laboratory throughout the study period.

\section{Randomization}

Despite repeated education to participating physicians, random allocation was substantially neglected throughout the recruitment period. The number of patients allocated to pravastatin $(n=3,061)$ was much larger than that of the conventional treatment group $(n=2,579)$. As seen in the baseline characteristics (Fig. 1, Table 2), men with known coronary risk factors were more likely to be allocated to pravastatin treatment. Serum total cholesterol levels were $13 \mathrm{mg} / \mathrm{dl}(0.34 \mathrm{mmol} / \mathrm{l})$ higher in the pravastatin group, and other lipid parameters were also significantly different between the two groups. The only exceptions were the prevalence of diabetes mellitus and smoking.

Nonpharmacological methods were used with 1,510 men $(59 \%)$ in the conventional treatment group. Because patients recruitment required a longer period than scheduled, the period of follow-up has exceeded 5 years for a proportion of the study subjects enrolled in the earliest phase. The study physicians are encouraged to continue the treatment and follow-up until the interim results are available.

\section{Statistical issues}

Statistical analysis on the endpoints was originally planned to be carried out on an intention-to-treat basis (irrespective of adherence to treatment) using a test of comparing proportions and the log-rank test. The failure in randomization, however, makes it inevitable to modify this principle. The Cox proportional hazard model will be used to control for covariates distributed differentially between the treatment groups as well as to allow for different times in follow-up arising from the delayed recruitment. Although the sample size was calculated with reference to the combined incidence of coronary events, similar analyses will be performed on not only cerebral infarction and total mortality but also on other long-term adverse outcomes such as cancer, cerebral hemorrhage, and suicide and accidental deaths. The occurrence of coronary events will also be analyzed in relation to the reduction in serum total cholesterol regardless of the type of treatment.

The rule of stopping the trial had not been a priori decided, and the overall balanced evidence for beneficial and adverse effects was to be evaluated once in the middle of follow-up with respect to continuation of the 


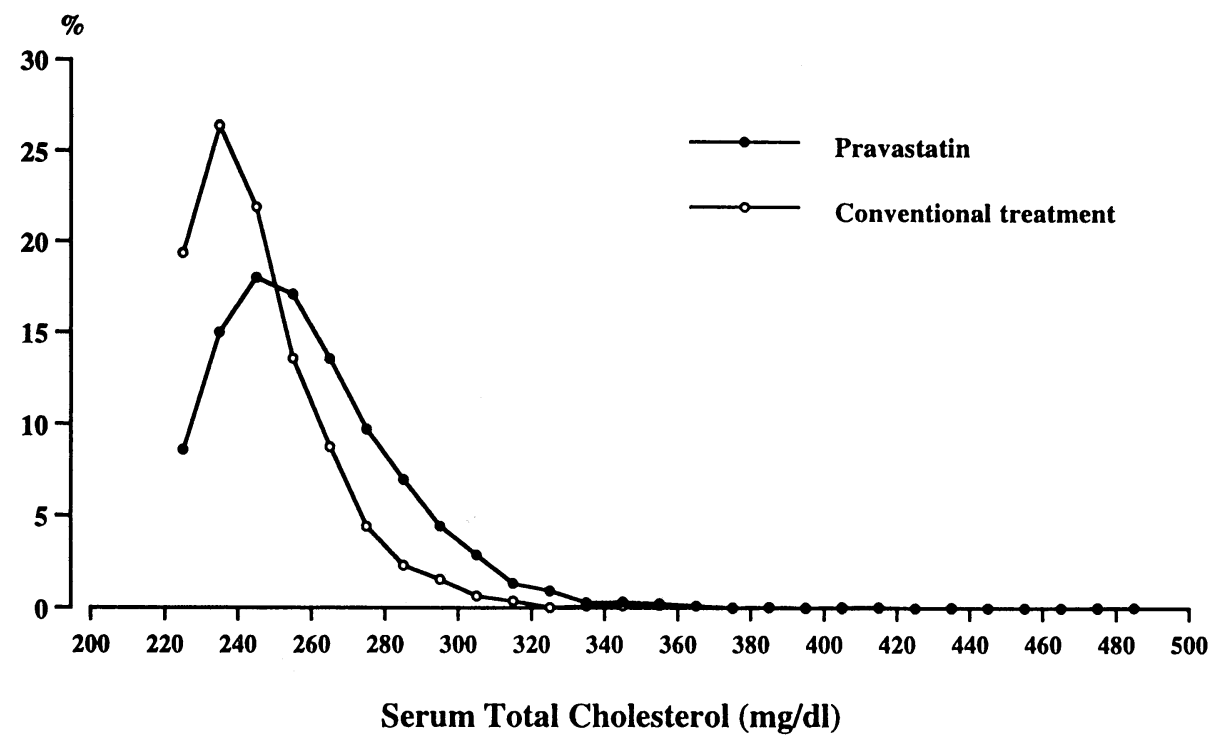

Fig. 1. Distributions of serum total cholesterol concentrations in the pravastatin and conventional treatment groups.

Table 2. Baseline characteristics of study subjects.

\begin{tabular}{lccc}
\hline \multicolumn{1}{c}{ Variable } & Pravastatin & $\begin{array}{c}\text { Conventional } \\
\text { treatment }\end{array}$ & P-value \\
\hline Number & 3061 & 2579 & \\
Age (year) & $57.8(7.9)$ & $57.3(8.3)$ & 0.02 \\
Body mass index $\left(\mathrm{kg} / \mathrm{m}^{2}\right)^{*}$ & $24.1(2.8)$ & $23.9(2.8)$ & 0.01 \\
Total cholesterol $(\mathrm{mg} / \mathrm{dl})$ & $258.7(25.9)$ & $245.5(20.2)$ & $<0.001$ \\
HDL cholesterol $(\mathrm{mg} / \mathrm{dll})^{*}$ & $48.7(12.1)$ & $49.4(12.0)$ & 0.03 \\
Triglycerides $(\mathrm{mg} / \mathrm{dl})^{* \dagger}$ & $179.0(1.7)$ & $161.7(1.7)$ & $<0.001$ \\
Systolic blood pressure $(\mathrm{mmHg})^{*}$ & $137.8(18.9)$ & $136.4(19.5)$ & 0.005 \\
Diastolic blood pressure $(\mathrm{mmHg})^{*}$ & $82.2(11.2)$ & $81.3(11.5)$ & 0.004 \\
Prior use of hypolipidemic drugs $(\%)$ & 13.7 & 8.0 & $<0.001$ \\
Use of antihypertensive drugs $(\%)$ & 38.2 & 34.6 & 0.006 \\
Angina pectoris $(\%)$ & 10.5 & 6.9 & $<0.001$ \\
Diabetes mellitus $(\%)$ & 26.8 & 26.7 & 0.93 \\
Current smoker $(\%)$ & 37.1 & 37.9 & 0.55 \\
Daily alcohol use $(\%)^{\ddagger}$ & 32.4 & 35.1 & 0.04 \\
\hline Values aremeans $(\mathrm{SD})$ & if otherwis & \\
\hline
\end{tabular}

Values are means (SD) if otherwise specified. Conversion factors for the unit of $\mathrm{mmol} / \mathrm{dl}$ are 0.02586 for cholesterol and 0.1129 for triglycerides.

* Data were not obtained for different numbers of patients: $0.5 \%$ for body mass index to $6.7 \%$ for HDL cholesterol.

+ Getmetric mean (approximate SD).

$\ddagger 5+$ times per week.

trial. However, amendment was made regarding interim analysis after the publication of results in the West of Scotland Coronary Prevention Study (13). Interim analysis on primary endpoints was rescheduled to be carried out twice using the data of 2.5 and 3.5 years each on average in the follow-up. Furthermore, allowance is to be made for multiple comparison according to the Peto's method (22); p-value less than 0.001 (one-sided) was set for the difference in the occurrence of coronary events combined as a criterion for stopping the trial. Annual review of the adverse effects including total mortality will also open a discussion on the termination of the trial if any apparent excess side-effects are noted in either the pravastatin or conventional treatment group.

\section{Safety monitoring}

Each study physician is responsible for detection of adverse effects occurring in his/her patients, and tempo- 
rarily reduces, or even discontinues, the drug dosage when medically indicated. Signs, symptoms, abnormalities in laboratory data, and concurrent diseases are recorded on the periodic follow-up forms. Biochemical and hematological data are referred to the reference values at the laboratory used by each physician. Any serious clinical problems occurring between the times of periodic reporting are notified to the Trial Office. Such data are reviewed by the Endpoint and Adverse Effect Subcommittee to provide appropriate advice to the study physicians and to ascertain whether these medical problems have been adequately addressed. The role of the Endpoint and Adverse Effect Subcommittee is adjunct to individual medical management of patients by the study physicians, however. Each item of laboratory and clinical data was graded in respect to the severity of adverse effects in accordance with the guidelines prepared by the Ministry of Health and Welfare.

The safety of the trial as a whole cannot be monitored adequately by individual study physicians. The External Review Committee systematically evaluates treatmentspecific aggregate data on endpoints, adverse symptoms, signs and diseases, clinical chemistry, compliance to treatment, withdrawal and lipid profile. Detailed statistical data for primary endpoints (coronary events), secondary endpoints (cerebral infarction and total mortality) and adverse effects are presented to the External Review Committee after the interim analyses. The External Review Committee also oversees general aspects concerning the conduct of the trial.

\section{Discussion}

The failure in randomization is a serious problem regarding the continuation of the trial. It may be argued that the trial is unethical because the scientific rigidity of a randomized trial has been lost. However, all the patients enrolled in the study are medically cared for by attending physicians with as much enthusiasm as paid to patients not participating in the study. Patients allocated to each group are treated for hypercholesterolemia with an officially approved regimen. Hypolipidemic drugs used in the conventional treatment group are less potent in reducing serum total cholesterol levels than pravastatin, but no study has addressed the benefit and safety of long-term use of pravastatin in Japan.

A recent publication of the Scottish trial showing an appreciable benefit of using pravastatin for hypercholesterolemia may raise another concern over continuation of the trial. The KLIS, however, is uniquely different from the Scottish trial in several important aspects. The subjects in the KLIS have lower levels of serum total cholesterol than those of the Scottish trial (mean $253 \mathrm{mg} /$ dl versus $272 \mathrm{mg} / \mathrm{dl}$ ); while a daily dose of $40 \mathrm{mg}$ was used in the Scottish trial, an approved dose of $10-20 \mathrm{mg}$ per day is used in the KLIS ; and patients in the control group also receive a currently approved medical treatment for hypercholesterolemia. Thus the KLIS will answer the question of practical importance as to whether low dosage of pravastatin is more beneficial in the management of mild or moderate hypercholesterolemia than conventional treatments. Furthermore, none of the previous cholesterol-lowering trials has examined specifically whether lowering blood cholesterol reduces the risk of cerebral infarction. Although the incidence rate of cerebral infarction was not a priori estimable, the occurrence of cerebral infarction is monitored as an endpoint to be studied. Since Japanese men seem to be at higher risk of cerebral infarction than Western populations (18), the KLIS will provide valuable information on lowering cholesterol and cerebral infarction. The KLIS is now regarded as a prospective observational study. Nevertheless the continuation of the study at least until the first interim analysis is justified because of the above-mentioned reasons.

There have been continuing concerns regarding the overall benefit of reducing cholesterol especially by drugs in the prevention of CHD events. Meta-analyses of intervention trials have drawn different conclusions (23-27). Those reluctant to use cholesterol-lowering drugs have recourse to the failure of establishing a benefit as regards total mortality and to an excess mortality from causes other than CHD, particularly accidental and violent death (23-25). On the other hand, Law et al. have concluded that reducing cholesterol even by drugs is not harmful at least in Western communities, arguing that total mortality is not an informative arbiter because of the limited statistical power in previous trials, individually or collectively, and that the reported excess mortality from causes other than CHD is spurious $(26,27)$. The Scottish trial showed no difference between the pravastatin and placebo groups in deaths from noncardiovascular causes combined, cancer, suicide, or trauma during a 5-year follow-up (13). Another trial of more than 4,000 patients with $\mathrm{CHD}$ in Scandinavia also found no difference in deaths from these causes between patients treated with simvastatin and those treated with a placebo (28). However, deaths from suicide and trauma were few in both studies: 11 in the Scottish trial (13) and 12 in the Scandinavian study (28). Serotonin metabolism has been implicated as being linked with violent behavior $(29,30)$, and a recent crosssectional study reported significantly lower plasma serotonin concentrations among men with chronically low cholesterol concentrations (31).

The KLIS was designed to detect a possible beneficial effect in terms of the incidence rate of $\mathrm{CHD}$ events combined, and thus the trial may not be sufficient statistically to address the effect on noncardiovascular mortality and morbidity. However, the KLIS will add to data as regards the relation of lowering cholesterol to cancer and violent deaths.

Finally, the crude rate of coronary endpoints may be 
higher in the pravastatin group in the KLIS because known coronary risk factors were more prevalent in this group. It is thus emphasized that comprehensive adjustment for these confounding factors is mandatory in order to address the question as to whether pravastatin use is beneficial in preventing coronary events.

Acknowledgements: The KLIS Executive Committee is grateful to all participating physicians for their cooperation, and to the staff of Sankyo, Co. Ltd. for their technical support. The study was supported by a grant from Sankyo, Co. Ltd.

\section{References}

(1) The Pooling Project Research Group: Relationship of blood pressure, serum cholesterol, smoking habit, relative weight and ECG abnormalities to major coronary events : final report of the Pooling Project. J Chronic Dis, 31: 201-306, 1978

(2) Pocock SJ, Shaper AG, and Phillips AN : Concentrations of high density lipoprotein cholesterol, triglycerides, and total cholesterol in ischemic heart disease. BMJ, 298 : 998-1002, 1989

(3) Rosengren A, Welin L, Tsipogianni A, and Wilhelmsen L: Impact of cardiovascular risk factors on coronary heart disease and mortality among middle aged diabetic men : A general population study. BMJ, 299: 1127-1131, 1989

(4) Stamler J, Wentworth D, and Neaton JD for the MRFIT Research Group: Is relationship between serum cholesterol and risk of premature death from coronary heart disease continuous or graded? Findings in 356, 222 primary screenees of the Multiple Risk Factor Intervention Trial (MRFIT). JAMA, 256: 2823-2828, 1986

(5) Committee of Principal Investigators: A co-operative trial in the primary prevention of ischemic heart disease using clofibrate. Br Heart J, 40: 1069-1118, 1978

(6) Committee of Principal Investigators: W.H.O. co-operative trial on primary prevention of ischemic heart disease using clofibrate to lower serum cholesterol: Mortality follow-up. Lancet, ii : 379-385, 1980

(7) Lipid Research Clinics Program: The Lipid Research Clinics Coronary Primary Prevention Trial results. I. Reduction in incidence of coronary heart disease. JAMA, 251 : 351-364, 1984

(8) Lipid Research Clinics Program: The Lipid Research Clinics Coronary Primary Prevention Trial results. II. The relationship of reduction in incidence of coronary heart disease to cholesterol lowering. JAMA, 251: 365-374, 1984

(9) Frick MH, Elo O, Haapa K, Heinonen OP, Heinsalmi P, Helo $P$, Huttunen JK, Kaitaniemi $P$, Koskinen $P$, Manninen V, Maenpaa $\mathrm{H}$, Malkonen M, Manttari M, Norola S, Pasternack A, Pikkarainen J, Romo M, Sjoblom T, and Nikkila EA : Helsinki Heart Study: Primary-prevention trial with gemfibrozil in middle-aged men with dyslipidemia. Safety of treatment, changes in risk factors, and incidence of coronary heart disease. N Engl J Med, 317: 1237-1245, 1987

(10) Szatrowski TP, Peterson AV Jr, Shimizu Y, Prentice RL, Mason MW, Fukunaga $\mathrm{Y}$, and Kato $\mathrm{H}$ : Serum choles- terol, other risk factors, and cardiovascular disease in a Japanese cohort. J Chronic Dis, 37 : 569-584, 1984

(11) Tsujita $Y$ and Watanabe $Y$ : Pravastatin sodium: A novel cholesterol-lowering agent that inhibits HMG-CoA reductase. Cardiovasc Drug Rev, 7: 110-126, 1989

(12) Saito $Y$, Goto $Y$, Nakaya N, Hata $Y$, Homma $Y$, Naito C, Hayashi H, Ito H, Yamamoto M, Takeuchi I, Mori K, Hara T, Yoshida S, Shirai K, Sasaki N, Shinomiya M, Murano S, Morisaki N, Nishiide T, Kanzaki T, Watanabe N, and Ishikawa $\mathrm{T}$ : Dose-dependent hypolipidemic effect of an inhibitor of HMG-CoA reductase, pravastatin (CS-514), in hypercholesterolemic subjects: A double blind test. Atherosclerosis, 72 : 205-211, 1988

(13) Shepherd J, Cobbe SM, Ford I, Isles CG, Lorimer AR, Macfarlane PW, McKillop JH, and Packard CJ for the West of Scotland Coronary Prevention Study Group: Prevention of coronary heart disease with pravastatin in men with hypercholesterolemia. $\mathrm{N}$ Engl J Med, 333: 1301-1312, 1995

(14) Consensus Conference: J Jpn Atheroscler Soc, 15: 1109-1148, 1987

(15) Halperin M, Rogot E, Gurian J, and Ederer F : Sample size for medical trials with special reference to long-term therapy. J Chronic Dis, 21: 12-24, 1968

(16) Ministry of Health and Welfare, Japan: Vital statistics, 1985. Health and Welfare Statistics Association, Tokyo, 1987

(17) Ueshima H, Tatara $\mathrm{K}$, and Asakura S: Declining mortality from ischemic heart disease and changes in coronary risk factors in Japan, 1956-1980. Am J Epidemiol, 125 : 62-72, 1987

(18) Uemura K and Pisa Z: Trends in cardiovascular disease mortality in industrialized countries since 1950. World Health Stat Q, 41: 155-178, 1988

(19) Ministry of Health and Welfare, Japan: National statistics on patients (Kanja-chousa), 1987. Health and Welfare Statistics Association, Tokyo, 1989

(20) Shimamoto T, Komachi $Y$, Inada H, Doi M, Iso H, Sato S, Kitamura $A$, lida $M$, Konishi $M$, Nakanishi N, Terao A, Naito $\mathrm{Y}$, and Kojima S: Trends for coronary heart disease and stroke and their risk factors in Japan. Circulation, 79 : 503-515, 1989

(21) Nakamura $Y$ : Serum total cholesterol and HDL-cholesterol levels in a community population of Kasuya County in connection with age, obesity and blood pressure. Jpn J Public Health, 33: 73-80, 1986 (in Japanese)

(22) Geller NL and Pocock SJ: The consultant's forum. Interim analyses in randomized clinical trials : Ramifications and guidelines for practitioners. Biometrics, 43: 213-223, 1987

(23) Muldoon MF, Manuck SB, and Matthews KA : Lowering cholesterol concentrations and mortality: A quantitative review of primary prevention trials. BMJ, 301: 309-314, 1990

(24) Ravnskov U: Cholesterol lowering trials in coronary heart disease: Frequency of citation and outcome. BMJ, 305: 15-19, 1992

(25) Smith GD, Song F, and Sheldon T : Cholesterol lowering and mortality: The importance of considering initial level of risk. BMJ, 306 : 1367-1373, 1993

(26) Law MR, Wald NJ, and Thompson SG: By how much and how quickly does reduction in serum cholesterol concentration lower risk of ischaemic heart disease. 
BMJ, 308: 367-372, 1994

(27) Law MR, Thompson SG, and Wald NJ : Assessing possible hazards of reducing serum cholesterol. BMJ, 308 : 373-379, 1994

(28) Scandinavian Simvastatin Survival Study Group: Randomized trial of cholesterol lowering in 4444 patients with coronary heart disease : the Scandinavian Simvastatin Survival Study (4S). Lancet, 344 : 1383-1389, 1994

(29) Engelberg $\mathrm{H}$ : Low serum cholesterol and suicide. Lancet, 339: 727-729, 1992

(30) Salter M : Low serum cholesterol and suicide. Lancet, 339: 1169, 1992

(31) Steegmans PHA, Fekkes D, Hoes AW, Bak AAA, van der Does $E$, and Grobbee DE: Low serum cholesterol concentration and serotonin metabolism in men. BMJ, 312 : 221, 1996

\section{Appendix 1. Membership of Committees}

Executive Committee*

Prof. Kikuo Arakawa (Chairman, Principal Investigator)

Second Department of Internal Medicine, Fukuoka University School of Medicine

Prof. Hajime Nawata (Trial Co-ordinator)

Third Department of Internal Medicine, Kyushu University

School of Medicine

Dr. Motosuke Hanada (Trial Co-ordinator)

Karatsu Red Cross Hospital

Dr. Yasushi Ishihara (Trial Co-ordinator)

Ishihara Cardiovascular Hospital

Dr. Jun Sasaki (Head of the Trial Office)

Second Department of Internal Medicine, Fukuoka University School of Medicine

Dr. Kei-ichi Araki (Head of Data Management Section)

Second Department of Internal Medicine, Fukuoka University School of Medicine

Dr. Masato Ageta (Endpoint and Adverse Effect Reviewer), Department of Internal Medicine, Miyazaki Nichinan Prefectural Hospital

Dr. Takashi Asano (Endpoint and Adverse Effect Reviewer)

First Department of Internal Medicine, Fukuoka University School of Medicine

Dr. Takehiko Fujino (Endpoint and Adverse Effect Reviewer)

Health Science Center, Kyushu University

Dr. Hisashi Kanaya (Endpoint and Adverse Effect Reviewer)

Kanaya Clinic, Fukuoka

Dr. Shunichi Koga (Endpoint and Adverse Effect Reviewer) Department of Internal Medicine, Ilzuka Hospital

Dr. Shiro Mawatari (Endpoint and Adverse Effect Reviewer)

Department of Health Science, Fukuoka Women's University

Prof. Takashi Yanagawa (Statistical Adviser)

Department of Mathematics, Kyushu University School of
Science

Prof. Suminori Kono (Trial Adviser)

Department of Public Health, Kyushu University School of Medicine

*The committee is also responsible for the writing of scientific papers.

\section{List of Local Organizers}

Fukuoka Prefecture : Dr. Sakutaro Takano (Takano Clinic), Dr. Masayuki Shimokobe (Kyoritsu Hospital), Dr. Yoichi Tanabe (Tanabe Clinic), Dr. Shigetada Ninomiya (Ninomiya Clinic), Dr. Mitsuo Fujino (Fujino Clinic), Dr. Keiichi Midorikawa (Midorikawa Cardiovascular Clinic), Dr. Yasushi Yokota (Yokota Hospital), Dr. Yoshihiko lkeda (Tagawa City Hospital), Dr. Fumio Umeda (Kyushu University Hospital), Dr. Hiroshi Kawashima (Omuta Rosai Hospital), Dr. Kazuyuki Saeki (Saeki Clinic).

Saga Prefecture: Prof. Toshiaki Sunaga (Saga Medical School), Dr. Kyosuke Yamamoto (Saga Medical School), Dr. Hideo Ikeda (Ikeda Clinic), Dr. Tadahiro Mizukami (Mizukami Clinic).

Oita Prefecture: Prof. Toshiie Sakata (Oita Medical School), Dr. Kanichiro Akioka (Akioka Clinic), Dr. Jin Iwao (Iwao Hospital), Dr. Yoshimi Ooshima, (Oshima Clinic), Dr. Yasuhiro Oribe (Mie Hospital), Dr. Toshio Goto (Goto Clinic), Dr. Susumu Shimada (Shimada Cardiovascular Clinic), Dr. Yoshihisa Shimazu (Simazu Clinic), Dr. Masashi Seita (Oita Red Cross Hospital), Dr. Masashi Tokieda (Tokieda Clinic), Dr. Nobuya Nagamatu (Saganoseki City Hospital), Dr. Ichiro Hata (Hata Hospital), Dr. Hideto Higashi (Higashi Clinic), Dr. Masaru Miyake (Miyake Clinic), Kohei Yamaguchi (Oita Prefectural Hospital).

Nagasaki Prefecture: Prof. Katsusuke Yano (Nagasaki University Hospital), Dr. Toshiyuki Imamura (Imamura Clinic), Dr. Yoshiyuki Miyahara (Nagasaki University Hospital), Dr. Fumiya Murakami (Murakami Clinic), Dr. Jiro Kubo (Kubo Clinic).

Kumamoto Prefecture : Prof. Motoaki Shichiri (Kumamoto University Hospital), Dr. Tomio Jinnouchi (Jinnouchi Hospital), Dr. Satoru Horita (Horita Cardiovascular Clinic), Dr. Takashi Honda, (Kumamoto Siseikai Hospital), Dr. Shoji Fukumitsu (Fukumitsu Cardiovascular Clinic), Dr. Takafumi Ohdo (Ohdo Clinic).

Miyazaki Prefecture: Dr. Takao Ayabe (Ayabe Clinic), Dr. Hiroshi Urakami (Urakami Clinic), Dr. Sumito Kariya (Kariya Clinic), Dr. Yasuhide Soda (Soda Hospital).

Kagoshima Prefecture: Prof. Hiromitsu Tanaka (Kagoshima University Hospital), Dr. Seiji Nishi (Nishi Cardiovasuclar Clinic), Dr. Takashi Tsuchimochi (Tsuchimochi Clinical).

\section{External Review Committee}

Prof. Yuichiro Goto (Chairman)

Tokai University School of Medicine, Kanagawa, Japan

Prof. Yuji Matsuzawa

Osaka University School of Medicine, Osaka, Japan 
Prof. Kazuo Ueda

Kyushu University School of Medical Technology, Fukuoka, Japan

Prof. Takesumi Yoshimura

University of Occupational and Environmental Health Sciences, Kitakyushu, Japan

Prof. lan Ford

Department of Statistics, Glasgow University, Glasgow, UK

\section{Appendix 2. Definitions of Coronary Endpoints}

1) Fatal or nonfatal myocardial infarction. Diagnosis of definite myocardial infarction requires one or more of the following categories:

(i) Autopsy findings of acute myocardial infarction.

(ii) Diagnostic ECG at the time of the event or at a periodic or unscheduled visit with no previous findings of myocardial infarction.

(iii) Severe substernal pain lasting for $30 \mathrm{~min}$ or longer plus either serum CPK elevation of at least twice the upper limit of the reference normal range or findings of coronary angiogram and ventriculogram compatible with myocardial infarction. The presence of "severe substernal pain lasting for $\mathbf{3 0}$ min or longer" alone is referred to suspect myocardial infarction, and is not enumerated as a primary endpoint.

2) Event of coronary artery bypass surgery or angioplasty.

3) Cardiac death - either (i) or (ii) category.

(i) Deaths occurring subsequently to symptoms or ECG findings suggestive of coronary heart disease (CHD).

(ii) Deaths occurring in those with known CHD when no cause other than CHD could be ascribed as the cause of death.

4) Sudden and unexpected death-all three are required:

(i) Deaths occurring within $1 \mathrm{hr}$ after the onset of severe symptoms or when last seen.

(ii) No known non-coronary acute or chronic process or event that was considered potentially lethal.

(iii) An 'unexpected' death is one that occurs only in a person who is not confined to his home, hospital, or other institution because of illness within $24 \mathrm{hr}$ before death.

\section{Appendix 3. Diagnostic Criteria} for Cerebral Infarction

Definite diagnosis of cerebral infarction requires both categories (i) and (ii).

(i) One or more of the following signs and symptoms lasting at least 24 hours: paresis or paralysis, speech dysfunction, unilateral sensory disturbance, disturbance of consciousness, dysphasia, visual disturbance, ataxia, or one or more spells of seizures (not necessarily lasting 24 hours).

(ii) Diagnostic findings in either computerized tomography $(\mathrm{CT})$ or magnetic resonance imaging (MRI) or cerebral angiography.

The presence of symptoms and signs alone is regarded as suspected cerebral infarction.

\section{Appendix 4. Estimations in Sample Size Calculation}

Several assumptions were needed to estimate the event rates in the study population during the study period. Firstly, the event rate among Japanese men aged 45-74 was estimated. Age-specific CHD death rates for Japanese men in 1990 and 1995 were estimated from the rates published in 1985 (16), assuming a 10\% reduction in each 5 years $(17,18)$. Assuming that patients enrolled in the study would have the same distribution in age as the outpatients in the national survey in 1987 (19), the mean annual death rate of $\mathrm{CHD}$ was estimated to be 1.27 per 1,000 in the next five years among Japanese men aged 45-74 years. Non-fatal CHD events were assumed to be twice as frequent as fatal CHD events, giving an annual event rate of 3.81 per 1,000 .

It was also assumed that serum total cholesterol concentrations were normally distributed with a mean of 190 and standard deviation of $35 \mathrm{mg} / \mathrm{dl}$ in Japanese men aged $45-74$ years $(20,21)$, and the proportions of men with serum total cholesterol of $<180,180-219$, and $\leq 220 \mathrm{mg} /$ dl were calculated to be $39 \%, 42 \%$ and $19 \%$, respectively. Relative risks of CHD events for men in these three categories of serum total cholesterol were assumed to be $1.0,1.5$ and 3.0 , respectively. In a Japanese prospective study over a period from 1958 to 1974 (10), relative risks of CHD incidence according to serum cholesterol levels were ; $<150 \mathrm{mg} / \mathrm{dl}: 1.0$ (referent), $150-180 \mathrm{mg} / \mathrm{dl}: 1.3$, 180-220 mg/dl : 1.9 and $>220 \mathrm{mg} / \mathrm{dl}: 2.2$. Relative risks of CHD mortality in the Multiple Risk Factor Intervention Trial were ; $<182 \mathrm{mg} / \mathrm{dl}: 1.0$ (referent), $182-220 \mathrm{mg} /$ $\mathrm{dl}: 1.5$ and $>220 \mathrm{mg} / \mathrm{dl}: 2.8$ (4). Since patients in the present trial are those visiting physicians due to medical reasons, they would tend to have clinical coronary risk factors such as hypertension, diabetes mellitus, and angina pectoris more frequently than the general population. Thus the relative risk of 3.0 for men with serum total cholesterol of $220 \mathrm{mg} / \mathrm{dl}$ or greater would be a reasonable assumption. From these data, the $\mathrm{CHD}$ event rate in patients recruited in the trial was expected to be 3.5\% for the five years without any treatment. 
Appendix 5. Clinical and Laboratory Data Collected at Baseline and in the Follow-up

Anthropometric variables : height (baseline only) and body weight.

Clinical measurements: pulse, systolic and diastolic blood pressure, chest $\mathrm{X}$-ray film and 12-lead electrocardiogram.

Serum lipids : total cholesterol, HDL cholesterol and triglycerides.
Biochemistry: total protein, total bilirubin, aspartate aminotransferase (AST), alanine amminotransferase (ALT), gamma-glutamyltransferase (GGT), Lactate dehydrogenase (LDH), alkaline phosphatase (ALP), creatinine phosphokinase (CPK), uric acid, urea nitrogen, creatinine and hemoglobin A1c.

Hematology : leukocyte count, erythrocyte count, hemoglobin and hematocrit.

Urine : qualitative test for sugar, protein and occult blood. 